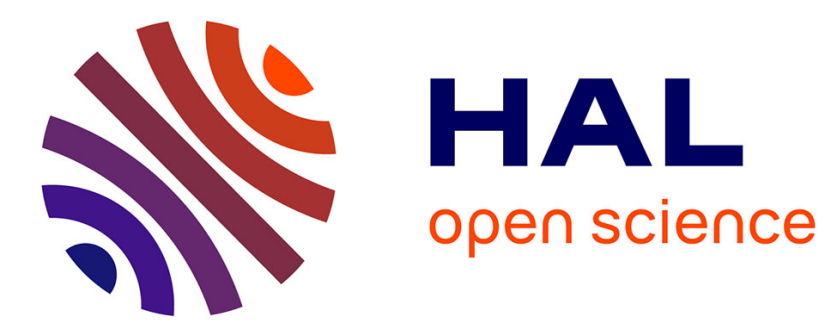

\title{
Polymer melt confined between two plates
}

Dominique Ausserré

\section{To cite this version:}

Dominique Ausserré. Polymer melt confined between two plates. Journal de Physique, 1989, 50 (19), pp.3021-3042. 10.1051/jphys:0198900500190302100 . jpa-00211121

\section{HAL Id: jpa-00211121 https://hal.science/jpa-00211121}

Submitted on 1 Jan 1989

HAL is a multi-disciplinary open access archive for the deposit and dissemination of scientific research documents, whether they are published or not. The documents may come from teaching and research institutions in France or abroad, or from public or private research centers.
L'archive ouverte pluridisciplinaire HAL, est destinée au dépôt et à la diffusion de documents scientifiques de niveau recherche, publiés ou non, émanant des établissements d'enseignement et de recherche français ou étrangers, des laboratoires publics ou privés. 
Classification

Physics Abstracts

$05.40-68.10-68.15-68.45$

\title{
Polymer melt confined between two plates
}

\author{
Dominique Ausserré \\ I.B.M. Research Division Almaden Research Center, 650 Harry Road, San Jose, California \\ 95120-6099, U.S.A. \\ and Institut Curie, Section de Physique et Chimie, 11 rue P. et M. Curie, 75005 Paris, France
}

(Reçu le 23 février 1989, révisé le 5 juin 1989, accepté le 8 juin 1989)

\begin{abstract}
Résumé. - On considère un fondu de polymères confiné entre deux plaques parallèles d'étendue finie et distantes de $D$. Le polymère est libre de s'échanger avec un réservoir de grandes dimensions. Lorsque $D$ est inférieure à la taille naturelle $R_{\mathrm{f}}$ des chaînes, ces dernières sont écrasées et le nombre de configurations qui leur sont accessibles est réduit. On doit alors associer à chaque chaîne une entropie de confinement $\Delta S_{1}$, dont on montre ici qu'elle varie avec $D$ comme $1 / D$. Comme le nombre $n$ de chaînes confinées est proportionnel à $D$, l'entropie de confinement totale $n . \Delta S_{1}$ du polymère piégé est indépendante de $D$ et la déformation des chaînes n'engendre aucune interaction à longue portée entre les plaques. La démonstration s'appuie sur une description détaillée de la configuration des chaînes confinées, qui apparaissent comme une succession de marches au hasard à trois et à deux dimensions. Leur distance bout à bout est fonction de $D$. Le cas de murs neutres impénétrables est discuté plus particulièrement. La configuration des chaînes polymériques est alors identique à celle des chaînes idéales en présence de deux parois adsorbantes lorsque l'énergie d'adsorption par monomère est l'énergie critique d'adsorption.
\end{abstract}

\begin{abstract}
A polymer melt confined between two parallel plates with a finite size and a distance $D$ between them is considered. The polymer is free to exchange with a bulk reservoir. When $D$ is smaller than the unperturbed size $R_{\mathrm{f}}$ of the chains, then they are squeezed and the number of their accessible configurations is reduced. Consequently, each of them experiences a confinement entropy loss $\Delta S_{1}$, which is shown to depend on $D$ as $1 / D$. Since the number $n$ of confined chains is proportional to $D$, the total entropy of confinement $n: \Delta S_{1}$ of the trapped polymer is independent of $D$. Therefore, the deformation of the chains does not induce any long range interaction between the plates. The demonstration is based on a detailed description of the configurations of the trapped chains, which appear as a succession of 2-dimensional and 3-dimensional random walks. Their end to end distance is a function of $D$. The case of impenetrable walls is thoroughly examined. Polymer configurations are then identical to those of ideal chains in presence of two adsorbing plates when the adsorption energy per monomer is the critical energy for adsorption.
\end{abstract}

\section{Introduction.}

The recent spreading of the surface force experiment as a commercial apparatus has allowed many physicists, biologists and chemists to explore structures and interactions in liquids at a quasi molecular level. In particular, the first studies of the interaction between two mica 
plates in presence of a polymer melt have been reported in the literature during the last three years $[1,2]$. The results obtained so far have been interpreted as an evidence of the existence of long range equilibrium repulsive forces between the two plates. This claim, and also the growing interest for thin polymer films in modern technologies, have recently motivated a lot of theoretical publications in the field [3-9]. The conclusion of most of them is that no force should be detected when thermodynamic equilibrium between the bulk reservoir and the polymer chains confined between the two plates is achieved. However, this conclusion has not yet been universally accepted probably due to the fact that it has not been understood in terms of configurations of the confined polymer chains. The aim of the present paper is to give a simple (probably simplified) physical picture allowing us to reach the same conclusion without detailed numerations nor abstract arguments. Our discussion will focus on the case where the interaction between the monomers and the wall is purely steric. However, we will also describe qualitatively the modifications we expect when the wall-monomer interaction is modified, or when pinning of the chains at the wall is introduced.

Far from any surface, polymer chains in the melt state are known to obey a Gaussian statistics identical to that of single ideal chains. As a consequence, it is tempting to identify the entropy variation $\Delta S_{1}$ associated with the transfer of one polymer coil from a bulk reservoir into the slit in both situations.

In the case of the single ideal chain trapped between two plates, $\Delta S_{1}$ has been derived by Asakura and Oosawa [10] and later by Casassa [11] using the analogy between trapped ideal chain configurations and trajectories of diffusing particles in the presence of absorbing walls. They found

$$
\Delta S_{1} \simeq-k_{\mathrm{B}}\left(\frac{R_{\mathrm{F}}}{D}\right)^{2} \quad \text { for } \quad a \ll D \ll R_{\mathrm{F}}
$$

where $k_{\mathrm{B}}, R_{\mathrm{F}}, a$ and $D$ hold for the Boltzmann constant, the Flory radius of the chain, the monomer size, and the distance between the plates.

Extending the use of this formula to the case of the polymer melt leads to the following development : «Confining the polymer chains requires $\Delta S_{1}$ per chain and $S_{1}$ scales with $D$ as $1 / D^{2}$. On the other hand, the number of confined chains in the slit is proportional to $D$, so that the total entropy of confinement is proportional to $1 / D$ and the plates repel each other ». An example is found in the calculation of John Barker [12] which has been used as a basis for interpreting recent results in the surface force experiment [1].

We clearly disagree with this model due to the following reason : the configurations of a single ideal chain and of a melt polymer chain, when trapped in the slit, are totally different.

Indeed, it has been shown by Asakura and Oosawa [10] that the consequence of confining ideal polymer chains between two neutral plates was a non-uniform distribution of the monomers in the slit, as shown in figure 1 by the qualitative shape $\rho(z)$. The profile is smooth, centered in the middle of the slit, and goes nearly to zero at the walls. If the statistics of the same chains in the melt state when confined between the same neutral plates were identical, then we would certainly get the same monomer distribution, i.e., the $\rho(z)$ profile. But the first constraint we have to respect when dealing with a melt is the requirement of a constant density as soon as the spatial scale under consideration is large compared to the monomer unit. It is therefore obvious that the chain configurations are not the same in both cases.

On the contrary, we will show that the entropy change required to confine the melt in the slit is not $\Delta S_{1} \simeq-k_{\mathrm{B}}\left(\frac{R_{\mathrm{F}}}{D}\right)^{2}$ per chain, but rather $\Delta S_{1} \simeq-k_{\mathrm{B}}\left(\frac{N a}{D}\right)$. Since the total number of chains in the slit is proportional to $D$, the total entropy of confinement does not 


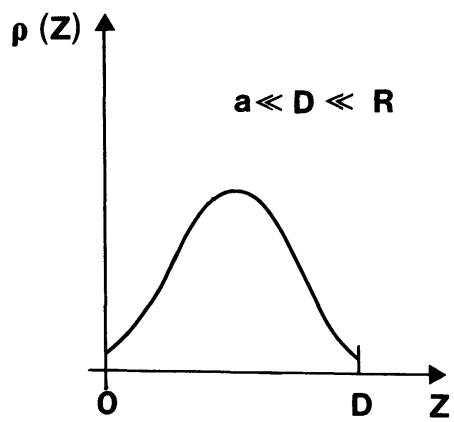

Fig. 1. - The qualitative shape of the monomer density profile $\rho(z)$ for a single ideal chain trapped between two plates when $a \ll D \ll R$.

depend on $D$, and no force is to be expected between the plates. Moreover, it will be explained in terms of chain configurations how the confinement entropy is localized in the very first surface layers, the structure of which is independent of $D$.

The way this work relates to the sum rule suggested by de Gennes [6] will finally be examined, and some consequences of our model will be drawn.

We will consider in the following that the polymer bonds are distributed on the cubic lattice shown in figure 2 with a mesh size a comparable to the statistical unit of the chain. Assuming that the polymer is fully flexible, $a$ is also the monomer size. The first and last « horizontal " layers of the lattice (001 planes) are lying on the walls, which distance $D$ corresponds to $D / a$ interlayers made of vertical segments. The height $z$ of any layer with respect to the lower wall will be related to its subscript $j$ by the relationship $j=z / a$. The subscripts of the two layers in contact with the solids are therefore $j=0$ and $j=D / a$. In the first part of this paper, we will consider the case of infinite chains. Chain ends will be introduced in the second part. Then, the entropy of confinement per chain will be derived in part 3 . In part 4, at last, we will show that the end to end distance of the chains is not independent of $D$ as it was supposed previously.

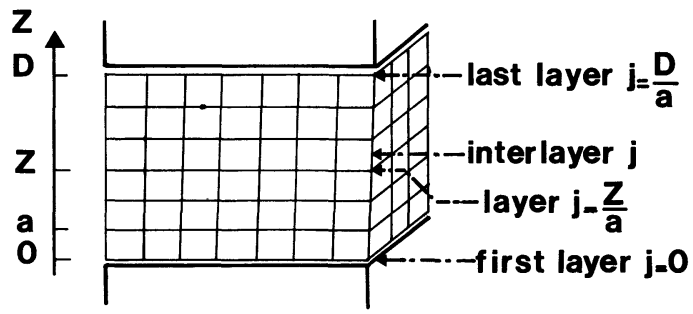

Fig. 2. - The cubic lattice used in the present paper. The polymer bonds are distributed over $j+1$ layers of lattice segments parallel to the walls and $j$ interlayers of lattice segments normal to the walls.

\section{Infinite chains.}

All segments parallel to the plates are sitting in horizontal layers, while segments normal to the plates are located in interlayers. This artificial distribution is due to the use of the lattice and it is obvious that the physical quantities considered in such a system have no meaning 
when averaged over distances comparable to or less than $a$. However, the choice of such a lattice with only parallel segments in contact with the wall is expected to be representative of a physical constraint on the statistical units [13-15]. The rate of occupancy of the lattice bonds by the polymer in the first $(z=0)$ and the last $(z=D)$ layers is not necessarily the same as in the bulk melt, but we expect the distribution to become uniform as soon as we leave them. In our schematic drawing, we will thus assume that the volume fraction is the bulk one everywhere else, including the first and the last interlayers. This is probably not strictly true because off-lattice Monte Carlo simulations have suggested the existence of oscillations for the density and the orientation order parameter of the bonds over two or three layers near the surfaces [3]. It would be possible to take care of this point by applying our arguments to the interlayers next to the perturbed region instead of the first ones. This would complicate very much the picture without bringing anything essential. So we will just ignore this difficulty.

Far from the plates, the monomer density is one. On the cubic lattice, the polymer bond density is, therefore, $1 / 3$, since there are 3 times as many bonds as monomers in the lattice. In agreement with the remarks above, we will thus assume that the polymer bond density is still $1 / 3$ in the first and the last interlayers. We will now check that it will not fix the segment density in the extreme layers. We have here to connect the vertical segments of the last interlayers by two-dimensional paths. If the segment density is the same at the surface as in the bulk, then $1 / 3$ of the lattice bonds has to be occupied. Since the maximum density for this two-dimensional sub-lattice is not $1 / 3$ as in all other layers, but $1 / 2$, it is possible to let it deviate from the bulk value over a wide range. On the other hand, there are many ways to tie two by two the $1 / 3$ of the lattice vertical bonds occupied by the polymer in the adjacent interlayers. By connecting nearest neighbors, we would minimize the path lengths in the surface layer, while we would increase it by connecting more distant monomers. This is illustrated in figure 3, where two very different sets of surface-paths are presented for connecting the same set of vertical polymer bonds in the adjacent interlayer.

As a consequence, we claim that the structure of the two extreme layers and the structure of all other layers and interlayers can be built up completely independently.

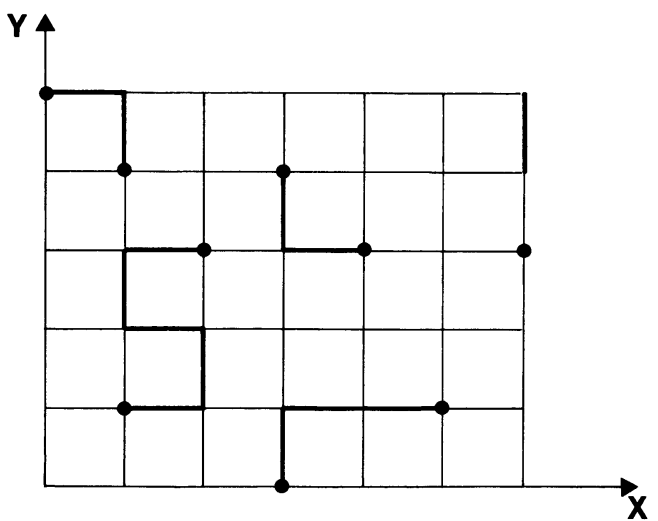

$3 a$ - short pathes

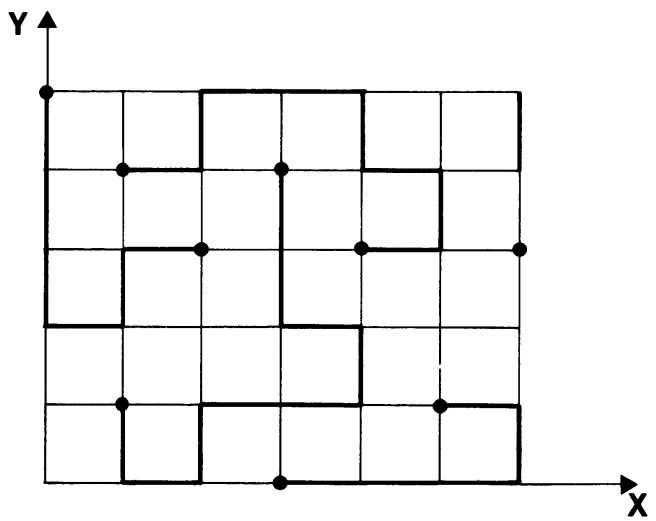

$3 b$ - Iorig-pathes

Fig. 3. - The surface layer, in the $X-Y$ plane. Dots are the projections of vertical segments in the last interlayers (or feet). Surface paths are drawn using solid lines. Figures $3 a$ and $3 b$ illustrate the cases of short and long 2D-paths. 
This argument is the key of the present paper. We can now consider that our system is made of two phases in thermodynamic equilibrium. The two layers on the walls will be referred to as the surface phase, and the remainder of the lattice as the bulk phase. In order to avoid confusion with the bulk phase made of unconstrained chains, we will refer to the latter as «the reservoir». Segments of the surface and bulk phases can exchange freely and the chemical potentials of both parts have to be equal. We can now depart from the restrictive implicit assumption of neutral plates : if the walls are repulsive, a chain arriving at the surface layer will tend to return to the bulk faster than if the walls were neutral ; on the contrary, the effect of an attractive wall will be to increase the 2D-path lengths and the monomer density at the surface. But in all cases, the number of 2D-paths will remain the same.

1.1 The BUlK STRUCTURE. - We will now focus on the structure of the polymer chains in the bulk phase. Because we restrict ourself to the case of infinite chains, we have simply to draw paths in the slit which connect two by two the bonds of the last two interlayers. These bonds will now be called the feet of the paths. Two kinds of paths have to be considered, those which connect two feet in the same extreme layer - we will call them loops - and those which connect two feet in the two different extreme interlayers - we will call them bridges. The terms loops and bridges (or ties) have been borrowed from the literature on semicrystalline polymers [16-18]. The main feature of the slit paths is obviously that they start and end at the walls, like doubly grafted polymers. On the other hand, their length is completely free of any constraint - which is an unusual property in polymer problems. The second requirement for the whole set of bulk paths is that they have to add up to a constant (one) density everywhere. Since it is possible to do so by considering only random paths for loops and bridges, it has to be the correct picture because it corresponds to a maximum entropy (or minimum energy) for the bulk phase. Any other class of paths will indeed require some additional constraint on the segment distribution, like anisotropy of the distribution or structural order in the succession of the segments along the chain or in space (this is actually a general rule). Because of the strict analogy between the amorphous phase of the semicrystalline polymer and the bulk phase in our problem, we could directly use the results of D Marzio et al. [16, 17]. However, we have chosen to justify here all the relations we need in view of giving a complete picture to the reader who is not familiar with the semi-crystalline system. From here, those who are only interested in arguments concerning the forces between plates may read part 3 independently.

1.1.1 Bridges. - A bridge is a random walk between levels $z=0$ and $z=D$. Its structure is invariant by a magnification $x \alpha$ affecting equally all directions in space, see figure 4 .

The magnification changes the $\mathrm{B}$ bonds of the bridge into $\mathrm{B}$ blobs of end to end distance $\alpha a$.

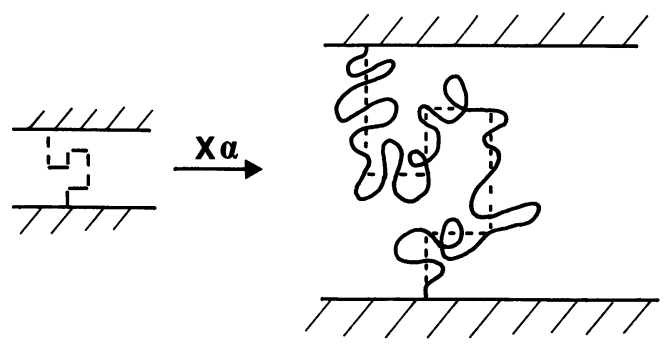

Fig. 4. - The magnification $X \boldsymbol{\alpha}$ used in part 1.1.1. 
Each blob is a random walk made of $\alpha^{2}$ bonds of size $a$. The scale change makes, therefore, the density decrease by a factor $1 / \alpha$, so that each path has to be repeated $\alpha$ times to get back the one density of the melt. The number of bridges in the whole system has now been multiplied by $\alpha$. Since the surface area of the walls has been magnified by a factor $\alpha^{2}$, the number of bridges per surface unit has been multiplied by $1 / \alpha$. The average length of a bridge has been changed by a factor $\alpha^{2}$. Hence we can write :

$$
L_{\mathrm{B}}=K_{1} \frac{D^{2}}{a} ; \quad \text { and } \quad n_{\mathrm{B}}=K_{2} \frac{1}{a D}
$$

denoting by $L_{\mathrm{B}}$ the average contour length of a bridge and by $n_{\mathrm{B}}$ the average number of bridges per surface unit. The constants $K_{1}$ and $K_{2}$ are determined by considering $D=a$, for which we shall get $L_{\mathrm{B}}=a$ and $n_{\mathrm{B}}=1 / 3 a D$. This sets $K_{1}$ to 1 and $K_{2}$ to $1 / 3$, and we finally obtain :

$$
L_{\mathrm{B}}=\frac{D^{2}}{a}
$$

and

$$
n_{\mathrm{B}}=\frac{1}{3 a D}
$$

1.1.2 Loops. - The number $n_{\mathrm{p}}$ of 3D-paths per surface unit is constant and equals

$$
n_{\mathrm{p}}=\frac{1}{3 a^{2}}
$$

The number of loops $n_{\mathrm{L}}$ per surface unit is, therefore :

$$
n_{\mathrm{L}}=\frac{D-a}{3 a^{2} D}
$$

and the fractions $f_{\mathrm{B}}$ and $f_{\mathrm{L}}$ of bridges and loops among 3D-paths are:

$$
f_{\mathrm{B}}=\frac{a}{D}
$$

and

$$
f_{\mathrm{L}}=1-\frac{a}{D}
$$

The numbers of segments $m_{\mathrm{B}}$ located in bridges is, per surface unit :

$$
m_{\mathrm{B}}=n_{\mathrm{B}} \frac{L_{\mathrm{B}}}{a}=\frac{D}{3 a^{3}} .
$$

And the total number of segments $m_{\mathrm{p}}$ in 3D-paths per surface unit is :

$$
m_{\mathrm{p}}=\frac{1}{a^{2}}\left(\frac{D}{3 a}+\frac{2(D-a)}{3 a}\right)=\frac{3 D-2 a}{3 a^{3}} .
$$

So that the total number of segments $m_{\mathrm{L}}$ in loops per surface unit follows to be :

$$
m_{\mathrm{L}}=\frac{2(D-a)}{3 a^{3}} \text {. }
$$


It is amazing to observe that the total number of horizontal and vertical bonds per surface unit are just, respectively, $m_{\mathrm{L}}$ and $m_{\mathrm{B}}$. Finally, the average length $L_{\mathrm{L}}$ of a loop is

$$
L_{\mathrm{L}}=\frac{2(D-a) a}{3 a^{3}} \cdot \frac{3 a^{2} D}{(D-a)}
$$

i.e. :

$$
L_{\mathrm{L}}=2 D
$$

At this point we have reached all the classical results of the gambler's ruin problem that we need in the following. We will now complete the description of the 3D-paths by deriving the monomer distribution in bridges and loops, and the distribution of loop lengths.

1.1.3 Distribution of monomers in bridges. - Following the picture of figure 5, we can divide a bridge into three parts for each arbitrary level $z$. Part $\mathrm{I}$ is a bridge between levels 0 and $z$. Starting from the surface, it stops the first time the chain meets level $z$. Part II then begins and ends the last time level $z$ is reached before going towards the other wall at level $D$. This third part is a bridge between levels $z$ and $D$. From the preceding section, we get :

- the average length of part I :

$$
L_{\mathrm{I}}(z)=\frac{z^{2}}{a}
$$

- the average length of part III :

$$
L_{\mathrm{III}}(z)=\frac{(D-z)^{2}}{a}
$$

- the average length of part II :

$$
L_{\mathrm{II}}(z)=L_{\mathrm{B}}-\left(L_{\mathrm{I}}(z)+L_{\mathrm{III}}(z)\right)
$$

i.e. :

$$
L_{\mathrm{II}}(z)=\frac{2 z(D-z)}{a} .
$$

Part II is made of an even number of loops starting from level $z$. Half of them extend below level $z$ without reaching the lower surface. Their average length is, therefore, $2 z$. Half of

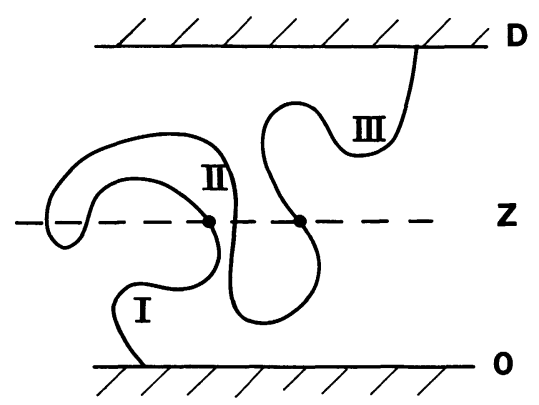

Fig. 5. - Any bridge can be divided at any level $z$ into three subchains I, II and III. Part I is a bridge between levels 0 and $z$. Part III is a bridge between levels $z$ and $D$. 
them extend towards the upper surface without reaching level $D$, and their average length is $2(D-z)$. The fraction $f_{\text {II low }}$ of the bonds of part II which are located below level $z$ is thus :

$$
f_{\text {II low }}(z)=\frac{z}{D}
$$

The total length of polymer $L_{(B \leqslant z)}$ below level $z$ then follows :

$$
L_{(B \leqslant z)}=L_{\mathrm{I}}(z)+f_{\mathrm{II} \mathrm{low}}(z) \cdot L_{\mathrm{II}}(z)=\frac{z^{2}}{a D}(3 D-2 z) .
$$

And the length of the chain between $z$ and $z+\mathrm{d} z$ is :

$$
\frac{\mathrm{d} L_{(B \leqslant z)}}{\mathrm{d} z}=\frac{6 z(D-z)}{a D} .
$$

The distribution of monomers in bridges $\frac{\mathrm{d} P^{(\mathrm{B})}}{\mathrm{d} z}(z)$, once normalized by $\int_{0}^{D} \frac{\mathrm{d} P^{(\mathrm{B})}}{\mathrm{d} z} \mathrm{~d} z=1$, is, therefore,

$$
\frac{\mathrm{d} P^{(\mathrm{B})}}{\mathrm{d} z}(z)=\frac{6 z(D-z)}{D^{3}} .
$$

The distribution is parabolic. For $z \ll D$, it becomes :

$$
\frac{\mathrm{d} P^{(\mathrm{B})}}{\mathrm{d} z}\left(\frac{z}{D} \rightarrow 0\right) \simeq \frac{6 z}{D^{2}}
$$

and appears as linear, which agrees with the profile predicted in reference 19 for single grafted chains in the presence of a melt.

1.1.4 Distribution of loop extensions and loop lengths. - A loop attached to the lower wall $(z=0)$ has a probability $w(\zeta)$ to be completely located below level $z=\zeta$ and a probability $\mathrm{d} w(\zeta)$ to reach exactly as an upper level layer $z$ such as $\zeta-\mathrm{d} \zeta \leqslant z<\zeta$. Its contour length is :

$$
s=\frac{2 \zeta^{2}}{a}
$$

since it is made of two bridges connected at level $z$. This is illustrated in figure 6 . Knowing that the average length of a loop is $2 D$, we get :

$$
2 \int_{0}^{D} \frac{\mathrm{d} w}{\mathrm{~d} \zeta} \frac{\zeta^{2}}{a} \mathrm{~d} \zeta=2 D
$$

which leads :

$$
\frac{\mathrm{d} w}{\mathrm{~d} \zeta}(\zeta)=\frac{a}{\zeta^{2}} \quad \text { (without cut-off) }
$$

This distribution will actually not diverge at small $\zeta$ because of the finite size $a$ of the bonds. Normalizing by :

$$
\int_{a}^{D} \frac{\mathrm{d} w}{\mathrm{~d} \zeta} \mathrm{d} \zeta=1
$$




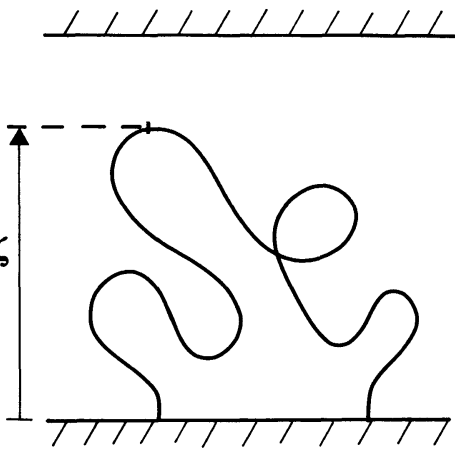

Fig. 6. - A loop of extension $\zeta$ is made of two bridges between levels $z=0$ and $z=\zeta$.

we obtain :

$$
\frac{\mathrm{d} w}{\mathrm{~d} \zeta}=\frac{1}{\left(1-\frac{a}{D}\right)} \frac{a}{\zeta^{2}}
$$

The distribution $\frac{\mathrm{d} P^{(\mathrm{CL})}}{\mathrm{d} s}(s)$ of loop contour lengths will also be useful. From (13) and (14), we get :

$$
\frac{\mathrm{dP}^{(\mathrm{CL})}}{\mathrm{d} s}(s)=\left(\frac{a}{2}\right)^{1 / 2} \cdot \frac{1}{\left(1-\frac{a}{D}\right)} s^{-3 / 2} .
$$

Using again the fact that a loop of extension $\zeta$ is made of two bridges between levels $z=0$ and $z=\zeta$, we get directly the distribution of monomers $\frac{\mathrm{d} P^{(L \zeta)}}{\mathrm{d} z}(z)$ in the loop by changing $D$ for $\zeta$ in relation (12) :

$$
\frac{\mathrm{d} P^{(L \zeta)}}{\mathrm{d} z}(z)=\frac{6 z(\zeta-z)}{\zeta^{3}}
$$

which verifies :

$$
\int_{0}^{\zeta} \frac{\mathrm{d} P^{(L \zeta)}}{\mathrm{d} z}(z) \mathrm{d} z=1 .
$$

To know that a bond of the bulk phase is part of a loop is not very informative if we do not know which surface the loop is attached to, and the picture would be by far incomplete if we were not deriving the concentration profile $\frac{\mathrm{d} m^{(\mathrm{L})}}{\mathrm{d} z}$ obtained by adding the contribution of all the loops attached to one of the two surfaces. The number of loops $\mathrm{d} n_{\mathrm{L}}$ extending up to $z$ as $\zeta-\mathrm{d} \zeta \leqslant z<\zeta$ is, per surface unit :

$$
\mathrm{d} n_{\mathrm{L}}(\zeta)=n_{\mathrm{L}} \frac{\mathrm{d} w}{\mathrm{~d} \zeta} \mathrm{d} \zeta=\frac{\mathrm{d} \zeta}{6 a \zeta^{2}}
$$


Relationship (17), obtained from (4) and (14), presents a remarkable feature : $\mathrm{d} n_{\mathrm{L}}(\zeta)$ does not depend on the distance $D$ between the plates. Only the loops extending up to $\zeta \geqslant z$ contribute to the concentration profile at height $z$, and their contribution is $\frac{\mathrm{d} P(\mathrm{~L} \zeta)}{\mathrm{d} z}(z)$ weighted by their length $s$, so that :

$$
\frac{\mathrm{d} m^{(L)}}{\mathrm{d} z}(z)=\int_{\zeta=z}^{D} \frac{\mathrm{d} \zeta}{6 a \zeta^{2}} \cdot \frac{6 z(\zeta-z)}{\zeta^{3}} \cdot \frac{2 \zeta^{2}}{a} .
$$

The number of bonds $\mathrm{d} m^{(\mathrm{L})}(z)$ at height $z$ per surface unit is, therefore :

$$
\mathrm{d} m^{(\mathrm{L})}(z)=\frac{1}{a^{3}} \cdot\left(1-\frac{z}{D}\right)^{2} \mathrm{~d} z .
$$

Figure 7 summarizes the partition of bonds in bridges and loops attached to each wall. It concludes the study of the bulk structure for infinite chains.

We will now concentrate on the second kind of connections between the vertical bond lying in the two extreme interlayers, the surface paths.

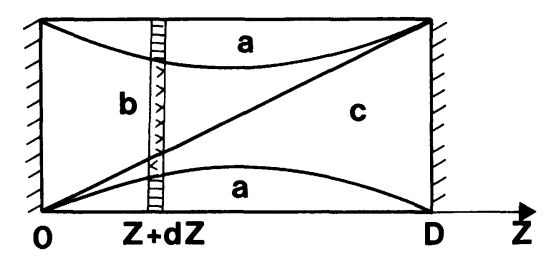

Fig. 7. - Diagrammatic representation of the monomer distributions in bridges (a) and loops connected to lower (b) and upper (c) walls. Between $z$ and $z+\mathrm{d} z$, the number of monomers in a, b, c are proportional to the areas dashed by resp.,$->$, and $<$ symbols. Area a has been split into parts in order to use the symmetry of the distributions with respect to $z=D / 2$.

1.2 THE SURFACE LAYERS. - The surface layer is made of $n_{2}$ paths of average length $\ell_{2}$ per surface unit. Since each path connects two bonds of the adjacent interlayer, we have :

$$
n_{2}=\frac{1}{6 a^{2}}
$$

The total number of bonds per surface unit is $\frac{n_{2} \ell_{2}}{6 a^{3}}$. It should not be confused with the total number of beads per surface unit. Since each path contains $\frac{\ell_{2}}{a}+1$ beads, the latter is $\frac{n_{2}}{6 a^{2}}\left(1+\frac{\ell_{2}}{a}\right)$. The fraction $\phi_{2}^{(\mathrm{m})}$ of the lattice sites occupied by beads (or monomers) and the fraction $\phi_{2}^{(b)}$ of the lattice segments occupied by polymer bonds are, therefore, different :

$$
\phi_{2}^{(\mathrm{m})}=\frac{n_{2} \ell_{2}}{6 a}\left(1+\frac{a}{\ell_{2}}\right)
$$


and

$$
\phi_{2}^{(b)}=\frac{n_{2} \ell_{2}}{6 a}
$$

This distinction is important because the structure of the surface layer will now depend on the elementary interactions acting on the monomeric units. As an example, it is clearly not equivalent to set an interaction between bonds or between monomers. It is also different to make the bonds or the monomers interact with the walls. It seems that only little attention has been paid to this point in this literature. We will emphasize it by comparing the results coming out of three different basic assumptions :

(i) In the first case, we impose the constraint of a full occupancy of the lattice sites by polymer beads in the surface layer (like in the bulk). Then we have $\phi_{2}^{(\mathrm{m})}=1$ and, therefore, $\phi_{2}^{(b)}=5 / 12$. The rate of occupancy of the lattice segments is much higher than in the bulk. As a consequence, the average 2D-path length turns out from (19), (20) to be $\ell_{2}=5$.

(ii) In the second case, we examine the consequence of imposing the same bond density in the surface layer as in a bulk horizontal layer, that is to say $\phi_{2}^{(b)}=1 / 3$. Then we get $\phi_{2}^{(m)}=5 / 6$. A depletion of the bead density appears at the wall. On the other hand, the average length of a $2 \mathrm{D}$-path is now $\ell_{2}=4$.

(iii) Instead of setting a requirement on the density of beads or bonds in the surface layer, we can also determine its structure by putting other kinds of constraints together with equations (19) and (20). As an example, we can force the segments to follow the rule of surface entropy reduction related to the change of the lattice coordination number at the surface [13] : when the path of a chain reaches the surface by occupying a segment in the last interlayer, it necessarily implies that the next segment, i.e., the first bond of the 2D-path, lies in the surface layer. The next step has 3 possible orientations in the surface layer and 1 towards the bulk. The probability of the surface path to reach a length $\ell$ is, therefore : $(1 / 4) \cdot(3 / 4)^{(\ell / a)-1}$, and the average length of a 2D-path is $\ell_{2}=\sum_{\ell / a=1}^{\infty} \ell \cdot P(\ell)=4 a$. As a consequence, the monomer and bond densities turn out to be $\phi_{2}^{(\mathrm{m})}=5 / 6$, and $\phi_{2}^{(\mathrm{b})}=1 / 3$, respectively. The surface entropy reduction rule leads, therefore, to the same results as example (ii) above.

A more « realistic» lattice model would contain an interaction energy term between monomeric units. This interaction insures the cohesion of the melt and also prevents very high densities to be reached in the vicinity of the wall. In other words, it guarantees that the deviation from the bulk density is not too large in the surface layer. It is not obvious, however, whether this elementary interaction should be defined as an interaction between bonds or between beads.

The examples above show that the choice of an elementary interaction between first neighbour bonds leads to the identity of the one density constraint for bonds and the surface entropy reduction rule. On the contrary, the choice of an elementary interaction between first neighbour beads would tend to set the monomer density in the first layer to one and a compromise would appear between this attempt and the trend for segments to reach the ideal situation of example (ii). Then, it is expected that the exact balance would be obtained for some surface path length intermediate between 4 and 5 units.

Considering that example (ii) (or equivalently example (iii)) offers a very satisfactory model for the case of the neutral impenetrable wall where no long-range interaction is able to modify the ideal structure found for the surface layer, we will now confine our study to this 
case and derive some simple additional results. We will first compare the quantities $n_{2}$ and $\ell_{2}$ to the equivalent quantities $n_{3}$ and $\ell_{3}$ defined in the bulk phase.

A $3 \mathrm{D}$-path is made of single vertical segments (interlayers) connected by horizontal paths located in horizontal bulk layers. Let us, therefore, call respectively $\ell_{3}$ the length and $n_{3}$ the average number per surface unit of such subchains. Using standard calculations based on the geometry of the cubic lattice, we get the probability $P_{3 D}(\ell)$ for such a path to be of length $\ell$ :

$$
P_{3 D}(\ell)=\frac{8}{25} \cdot\left(\frac{3}{5}\right)^{\frac{\ell}{a}-1} \quad \text { for } \quad \ell \geqslant 0
$$

and the questioned average length is, therefore :

$$
\ell_{3}=\sum_{\ell=0}^{\infty} \ell \cdot P_{3 D}(\ell)=2
$$

the path of length 0 being obtained by connecting directly two vertical segments lying above and below the horizontal layer under consideration. So, we conclude :

$$
2 \cdot \ell_{3}=\ell_{2}
$$

and, consequently,

$$
n_{3}=2 n_{2}
$$

Each horizontal layer of the bulk phase is made of twice more 2D-paths than the surface layers, and each path contains twice less monomers.

We wish now to examine at a very rough level the effect of an interaction energy $U_{a}$ between the polymer bonds and the wall. For the sake of simplicity, we will assume the range of this interaction to be small when compared to $a$. The minimum value for $\ell_{2}$ is 1 , corresponding from (19) and (20) to $\phi_{2}^{(\mathrm{m})}=1 / 3$ and $\phi_{2}^{(\mathrm{b})}=1 / 2$. This corresponds to the highest repulsive potential $U_{a \max }$ compatible with our picture. The maximum value for $\ell_{2}$ is 5 . Then we get $\phi_{2}^{(\mathrm{m})}=1$ and $\phi_{2}^{(\mathrm{b})}=5 / 12$, which is the maximum rate of occupancy for bonds in the surface layer compatible with the $1 / 3$ density in the last interlayer. This case corresponds to the highest attractive interaction allowed within the present frame. In this case ve set $U_{a}=U_{a \min }(<0)$.

By increasing the wall monomer interaction above $U_{a \max }$, we will obviously affect the adjacent interlayer of vertical segments, and thus propagate the lack of bonds in the surface layer to upper levels.

By decreasing the wall monomer interaction below $U_{a \min }$, we will cross the upper threshold $\ell_{2}=5$ and increase the bond density towards the absolute limit $1 / 2$ corresponding to a complete ordering of the surface layer. From there, a lack of vertical bonds in the first interlayer follows, inducing a propagation of the wall effect towards the bulk as for $U_{a}>U_{a \max }$.

A naive estimation of the thresholds $U_{a \min }$ can be obtained by introducing a bias in the surface coordination number of the lattice. The probability for a bond to occupy a segment of the lattice surface layer has to be changed from $1 / 4$ (see example (ii) of this section) to $\frac{1}{1+3 \exp \left(-\frac{U_{a}}{k_{\mathrm{B}} T}\right)}$. Using the same calculation as we did for deriving $\ell_{2}$, we now get the 
average length $\ell_{2}\left(U_{a}\right)$ of the surface paths in the presence of the interaction potential $U_{a}$ :

$$
\ell_{2}\left(U_{a}\right)=1+3 \exp \left(-\frac{U_{a}}{k_{\mathrm{B}} T}\right)
$$

$U_{a}=0$ gives $\ell_{2}(0)=\ell_{2}=4$ as expected,

$U_{a}=+\infty$ gives $\ell_{2}=1$ as expected.

The maximum length $\ell_{2}=5$ of the path imposed by the $1 / 3$ constraint in the close interlayer corresponds to :

$$
\frac{U_{a \min }}{k_{\mathrm{B}} T}=\ln \frac{3}{4}
$$

which is numerically close to $U_{a \min }=-2 / 7 k_{\mathrm{B}} T$. The threshold $U_{a \min }$ is therefore very easy to reach. However, this analysis is extremely naive since any perturbation of the surface layer density gives rise to an additional surface energy term generated by the monomer-monomer interactions. The effect of this cohesion energy will obviously be to lower the threshold $U_{a \min }$. A complete description would therefore establish $U_{a \text { min }}$ by balancing the energy gained in filling the surface layer and the excess of local pressure induced by this increase in the bond density. What we want to qualitatively point out here is the fact that a high attractive energy between the bonds and the wall is able to introduce a strong order in the surface layer. A crystallization of the polymer at the surface might be the most direct consequence of this ordering.

In the above parts 1.1 , and 1.2 , we have considered the length of the polymer chains as infinite. This approximation is valid in practice when the surface of the plates is smaller than the squared Flory radius of the unperturbed chains, and for interplate distance $D \ll R_{\mathrm{F}}$.

It is also expected to apply for cyclic polymers of length $N a \gg D$, whatever the surface area of the plates can be.

In order to deal with the more realistic case of linear homopolymers between large plates, we shall now examine the case of finite chains.

\section{Finite chains.}

In addition to the two kinds of 3D-paths mentioned in part 1, i.e. loops and bridges, we have to consider two other kinds of subchains : tails and free chains. Tails are connected once to one of the two walls, while free chains are not connected to the solid surfaces. Because the two types of new paths have at least one free end, we will call both of them « open paths »By opposition loops and bridges will be classified as «close paths ». The presence of open paths in the slit implies a reduction of the number of close paths. On the other hand, the distribution of monomers in the open paths and close paths is such that the one density constraint remains verified everywhere. Moreover, it is obvious that tails and free chains will, like loops and bridges, be simple random walks if this is compatible with all the requirements of our problem. We will show that it is the case by using a process for generating the open paths which respects these requirements. We call it the cutting process.

Starting from an infinite chain as shown in figure 8 , we choose randomly a monomer along the chain and then follow the contour length from that point, keeping always the same direction. Each time we have made a distance $\mathrm{Na}$, we cut the chain and go on our trip. The result of the process is a set of monodisperse polymer chains of length $\mathrm{Na}$. Some of the cuts are in the surface layer, and some of them are in the bulk phase. The cutting process has obviously removed some bridges if not all, and some loops. The open paths generated in this way are exactly random walks, and the one density constraint of the problem is still fulfilled. 


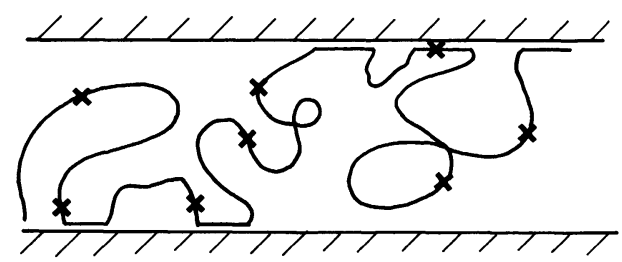

Fig. 8. - Illustration of the cutting process described in part 2. Cutting points are represented by crosses. The contour length between two successive cuts is always the same.

However, the open paths obtained in this way are facing their ends by pairs and this cannot correspond to a general picture for a melt confined between two plates. If we want to reach it, we still have to apply a mixing process to the ordered set of paths. This mixing is performed by exchanging the paths using translations in the $x y$ plane. It is important to realize that this last step will not affect the statistical distributions in the $z$-direction, like the monomer profile in tails or the distribution of tail extensions, nor it will affect either the quantities averaged over the surface, such as the number of tails of a given length per surface unit for example. The cutting process alone is thus expected to generate all the pertinent distributions. It should be noted that our cutting process is consistent with a model of paths. This mixing is performed by exchanging the paths using translations in the $x y$ plane. It is important to realize that this last step will not affect the statistical distributions in the $z$-direction, like the monomer profile in tails or the distribution of tail extensions, nor it will affect either the quantities averaged over the surface, such as the number of tails of a given length per surface unit for example. The cutting process alone is thus expected to generate all the pertinent distributions. It should be noted that our cutting process is consistent with a model of interacting bonds rather than interacting beads, since cutting a chain at a lattice vertex results in the superposition of two terminal beads at this point. With interacting beads on the contrary, it should be necessary to cut the chain between to lattice sites, i.e. to remove one polymer bond at each cut, and therefore to slightly modify the bond concentration. On the other hand, terminal monomers are always different from others as chemical units and do not interact with their surrounding in the same way. This effect has not been considered ; this is the main approximation involved in the cutting process.

2.1 Result OF THE CUTTING PROCESS. - In this section, we examine some features of the open paths generated by the cutting process. The starting picture is the system of infinite chains considered in the first part, where all the paths are closed. Calling $\ell$ the original length of such a path, we first derive the probability of getting $p$ cuts along the path as a function of $\ell$.

(i) If $0 \leqslant \ell<N a$, the probability $P_{1}(\ell)$ for cutting the path once is proportional to its length $\ell$ and also has to verify :

$$
\begin{aligned}
& P_{1}(\ell=0)=0 \\
& P_{1}(\ell=N a)=1
\end{aligned}
$$

This leads :

$$
P_{1}(\ell)=\ell / N a
$$

The probability $\overline{P_{1}(\ell)}$ that no cut was obtained is the complement of $P_{1}(\ell)$ :

$$
\overline{P_{1}(\ell)}=1-\frac{\ell}{N a} \text {. }
$$


(ii) If $N a \leqslant \ell<2 N a$, the probability $P_{2}(\ell)$ of cutting the path twice is :

$$
P_{2}(\ell)=\frac{\ell}{N a}-1
$$

while the probability of getting only one cut is :

$$
\overline{P_{2}(\ell)}=2-\frac{\ell}{N a} \text {. }
$$

Getting no cut is impossible.

(iii) Generalization to $(p-1) N a \leqslant \ell<p N a$ gives :

- the probability of getting $p$ cuts :

$$
P_{p}(\ell)=\frac{\ell}{N a}-p+1
$$

- the probability of getting $p-1$ cuts :

$$
\overline{P_{p}(\ell)}=p-\frac{\ell}{N a} \text {. }
$$

These results are illustrated in figure 9 where the probabilities of getting $p$ cuts are represented by $p$-lines.

As a direct application, we will now derive the total number of free chains per surface unit in the slit, which is a function of the interplate distance $D$.

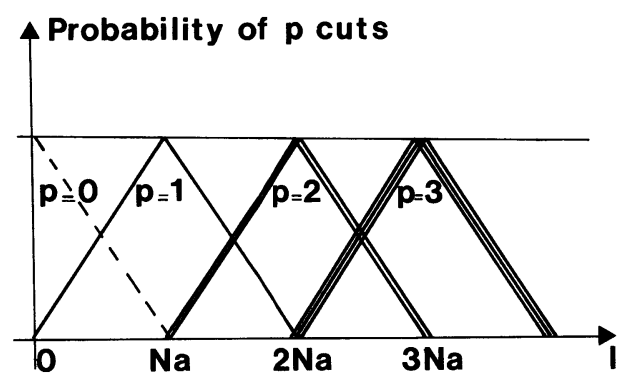

Fig. 9. - Result of the cutting process : probability of getting $p$-cuts in a bulk path as a function of its length $\ell$. Na is the contour length between cutting points. Fach $p$-line holds for $p$ cuts. The dotted line holds for $p=0$.

2.1.1 Free chains. - No free chain is coming out of the cutting process as long as the length $\ell$ of every original path is smaller than $\mathrm{Na}$. The longest closed-paths in the slit are loops of extension $\zeta=D$. Their contour length is $\ell_{\mathrm{M}}=\frac{2 D^{2}}{a}$. Therefore, there is no free chain in the slit if $\ell_{\mathrm{M}}<N a$, i.e. if $D<\frac{N^{1 / 2} a}{\sqrt{2}}$. We set $D_{\mathrm{M}}=\frac{N^{1 / 2} a}{\sqrt{2}}$.

Following the experiments performed on melts using the surface force apparatus, it has been suggested that the chains at the surface could be irreversibly pinned at the wall [7]. In such situations, a sharp transition in the force vs. $D$ curves should be observed at 
$D=D_{\mathrm{M}}$. Indeed, the threshold $D=D_{\mathrm{M}}$ would be the transition between a regime of thermodynamical equilibrium between the bulk slit and the reservoir for $D>D_{\mathrm{M}}$, and a regime where no chain can be exchanged between both phases for $D<D_{\mathrm{M}}$. In the latter, the slit has to be considered as a closed system in the thermodynamic sense. This probably explains the position of the observed «hard wall» at $D \simeq D_{\mathrm{M}}$ in the surface force experiments.

The average number of cuts $c(\ell)$ performed along a path of length $\ell$ such as $(p-1) N a \leqslant \ell<p N a$ is :

$$
c(\ell)=p P_{p}(\ell)+(p-1) \overline{P_{p}(\ell)}
$$

i.e.

$$
c(\ell)=\frac{\ell}{N a}
$$

which is independent of $p$.

We now consider separately the free chains generated from bridges and loops for a given distance $D>D_{\mathrm{M}}$ between the plates.

(i) Bridges : The number of cuts per bridge is $\frac{D^{2}}{N a^{2}}$. It leads directly to the number of free chains $F_{\mathrm{B}}(D)$ generated per bridge :

$$
\begin{array}{lll}
F_{\mathrm{B}}(D)=0 & \text { if } & D<N^{1 / 2} a \\
F_{\mathrm{B}}(D)=\frac{D^{2}}{N a^{2}}-1 & \text { if } & D \geqslant N^{1 / 2} a
\end{array}
$$

The present calculation neglects the fluctuations around the mean length $\frac{D^{2}}{a}$.

(ii) Loops : The number of cuts $c_{\mathrm{L}}(\zeta)$ per loop of extension $\zeta$ is :

$$
c_{\mathrm{L}}(\zeta)=\frac{2 \zeta^{2}}{N a^{2}}
$$

Since free chains are generated when the path is cut at least twice, only loops with extension $\zeta \geqslant \zeta_{\mathrm{M}}$ will contribute. $\zeta_{\mathrm{M}}$ is obtained from, $\frac{2 \zeta_{\mathrm{M}} 2}{a}=N a$, i.e. $\zeta_{\mathrm{M}}=\frac{N^{1 / 2} a}{\sqrt{2}}\left(=D_{\mathrm{M}}\right)$. On the other hand, the number of free chains $F(\ell)$ and the number of cuts $c(\ell)$ generated per path of length $\ell$ are related by:

$$
F(\ell)=c(\ell)-1 \quad \text { when } \quad c(\ell) \geqslant 1 .
$$

Using the distribution (14) of loop extensions, we can thus estimate the average number $F_{\mathrm{L}}(D)$ of free chains generated per loop in the slit of thickness $D>D_{\mathrm{M}}$ :

$$
F_{\mathrm{L}}(D)=\frac{\int_{\zeta_{\mathrm{M}}}^{D} \frac{a}{\zeta^{2}}\left(\frac{2 \zeta^{2}}{N a^{2}}-1\right) \mathrm{d} \zeta}{\int_{a}^{D} \frac{a}{\zeta^{2}} \mathrm{~d} \zeta}
$$


i.e. :

$$
F_{\mathrm{L}}(D)=\frac{1}{\left(1-\frac{a}{D}\right)}\left(D-\zeta_{\mathrm{M}}\right) \cdot\left(\frac{2}{N a}-\frac{a}{D \zeta_{M}}\right)
$$

(iii) Using relations (5) and (6) which give the fractions of bridges and loops among the original closed paths, we get the total number of free chains $F(D)$ generated per path in the cutting process as a function of $D$ :

a) For $\zeta_{\mathrm{M}}<D \leqslant N^{1 / 2} a$, only loops contribute, and :

$$
F(D)=\left(1-\frac{a}{D}\right) \cdot F_{\mathrm{L}}(D)
$$

i.e. :

$$
F(D)=\frac{2 D}{N a}+\frac{a}{D}-2 \sqrt{\frac{2}{N}}
$$

b) For $D \geqslant N^{1 / 2} a$ loops and bridges have to be considered, and :

$$
F(D)=\left(1-\frac{a}{D}\right) \cdot F_{\mathrm{L}}(D)+\frac{a}{D} \cdot F_{\mathrm{B}}(D) .
$$

From where we finally get :

$$
F(D)=\frac{3 D}{N a}-2 \sqrt{\frac{2}{N}} .
$$

The results are summarized below :

$$
\left\{\begin{array}{lll}
F(D)=0 & & a \ll D \leqslant \frac{N^{1 / 2} a}{\sqrt{2}} \\
F(D)=\frac{2 D}{N a}+\frac{a}{D}-2 \sqrt{\frac{2}{N}} & \frac{N^{1 / 2} a}{\sqrt{2}} \leqslant D \leqslant N^{1 / 2} a \\
F(D)=\frac{3}{N a} D-2 \sqrt{\frac{2}{N}} & N^{1 / 2} a \leqslant D .
\end{array}\right.
$$

The variations (24) of the number of free chains in the slit as a function of $D$ are displayed in figure 10. The discontinuity appearing in figure 9 would be removed by considering statistical fluctuations in the bridge lengths.

2.1.2 Tails. - Starting from a 3D-path of length $\ell$ such as $0 \leqslant \ell<N a$, the probability that two tails are created in the cutting process is $P_{1}(\ell)$. Their average length is $\ell / 2$. No tail appears if the path is not cut and the corresponding probability is $\overline{P_{1}(\ell)}$. Multiplying $F(D)$ by $n_{p}=1 / 3 a^{2}$ (Rel. (3)) gives the number of free chains per surface unit.

For 3D-paths of length $\ell$ such as $(p-1) N a \leqslant \ell<p N a$, where $p$ is an integer greater than 1 , the probability that two tails are generated is 1 . Adding their lengths gives $\ell-(p-1) N a$ with a probability $P_{p}(\ell)$ and $\ell-p N a$ with a probability $\overline{P_{p}(\ell)}$. The average length of each tail is therefore $\frac{N a}{2}$ for any $\ell \geqslant N a$.

These elements are summarized in figure 11. From there, it is straightforward to derive the full distribution of tail lengths. It is then possible to establish the monomer concentration 


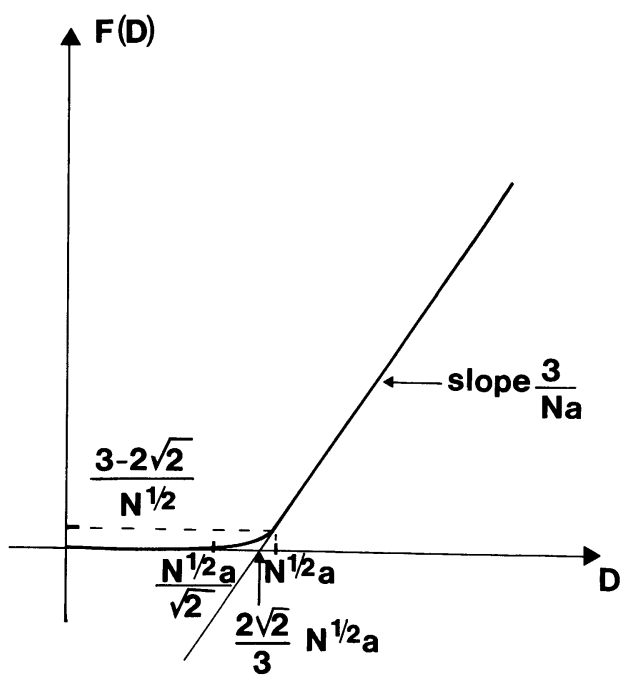

Fig. 10. - Number of free chains generated per path versus distance $D$ between plates.

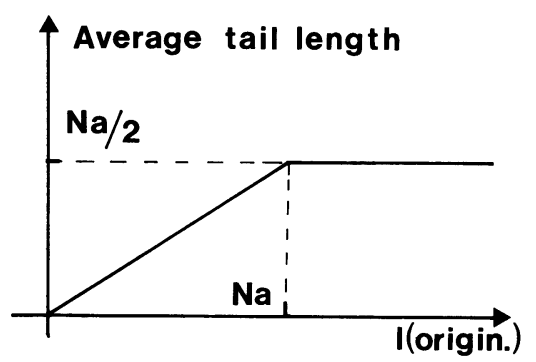

Fig. 11. - Average length of the tails generated by the cutting process as a function of the length $\ell$ of the original bulk paths.

profiles for tails and for chains connected to the wall. As a consequence, the monomer distribution for free chains, which exhibits a depletion layer, can also be obtained. These results, however, will not be presented in this paper.

\section{Entropy of confinement per chain.}

In the case where the distance $D$ is much smaller than the natural size $R_{\mathrm{F}}$ of the chains, each polymer molecule is made of a large number of loops and bridges obeying the previously derived distribution. The monomer distribution inside one chain is therefore a flat profile extending all over the bulk phase. The monomer concentration can depart from its bulk value in the surface layers if the plates are not neutral. But in all cases the number of segments per chain with a restricted orientation is proportional to the number of surface paths per chain. This is still true (in average) for finite chains and large distances between the plates. Since the bulk paths are simply random walks (as it would be for the same parts of the chain if they were transferred to the reservoir), they do not contribute to the confinement entropy per chain $\Delta S_{1}$, which is therefore simply proportional to the number of 2D-paths per chain. Since the 
total number of 2D-paths in the slit is independent of $D$, while the total number of chains in the slit is proportional to $D$, we get :

$$
\Delta S_{1}=-k_{\mathrm{B}} \beta\left(U_{a}\right)\left(\frac{N a}{D}\right)
$$

where $\beta\left(U_{a}\right)$ is a function of the wall-monomer interaction, but not of $D$. Note that $\Delta S_{1}$ is not a power law of $\left(\frac{R_{\mathrm{F}}}{D}\right)$.

As announced previously, $\Delta S_{1}$ turns out to be very different from the entropy loss experienced by single ideal chains trapped in the same geometry. This difference is not surprising when one realizes that the chain configurations have not the same structure in both problems. This is illustrated by figure 12. The melt configuration fills the space uniformly exactly as a Brownian particle would do when observed between the plates over long enough time scales (the analogy does not hold for the surface paths). The trapped ideal chain is not equivalent to the trapped Brownian flight [20]. Actually, its local structure (range greater than a and smaller than $D$ ) is not a free random walk, but is biased by the presence of the walls everywhere in the slit. For example, when the walk reaches any level $z<D / 2$, the probability for further steps to be directed upwards is higher than to be directer downwards. Since monomers of this (hypothetical) ideal chain experience no exclude volume with each other, the only physical origin of the long-range effect of the wall is the connectivity of the chain. By opposition, the connectivity is screened out in the case of the melt chain.

It is indeed very easy to modify the ideal chain problem in order to obtain an exact equivalence with the melt between neutral plates. For this purpose, it is necessary and sufficient to force the surface density of bonds to equal the bulk density. Equivalently, we have to force the length of the $2 \mathrm{D}$-paths to be the same as in the melt problem. This is achieved by introducing the proper attractive interaction energy $U_{a}=U_{a_{\mathrm{c}}}$ between the bonds and the wall. This is a classical polymer problem, the energy $U_{a_{\mathrm{c}}}$ being the so-called critical energy for adsorption. A review of this subject is presented in a recent work of Bouchaud [21].

Using the analogy, we can deduce that $U_{a}=U_{a_{\mathrm{c}}}$ in the ideal chain problem leads to a flat distribution of the polymer bonds in the ideal chain problem leads to a flat distribution of the polymer bonds in the slit. This conclusion is actually in perfect agreement with the work of Di
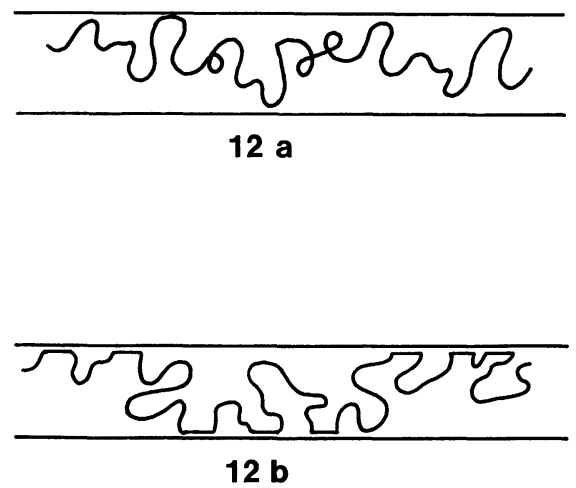

Fig. 12. - a) Isolated ideal chain between two neutral plates. b) One chain of the melt confined between two neutral plates. Also: isolated ideal chain between two adsorbing plates when $U a=U a_{\mathrm{c}}$. 
Marzio and Rubin [22]. In their study, the distance between the walls was small $\left(D \ll N^{1 / 2} a\right)$. It seems however riskless to generalize the flat distribution to higher distances $D$ between the plates. We therefore claim that the concentration profile obtained when confining a solution of ideal chains between two adsorbing plates is flat when $U_{a}=U_{a_{\mathrm{c}}}$ for any distance $D \gg a$. This conclusion holds for $D \rightarrow \infty$, i.e. for the single plate problem. We obviously include in the flat profile the bonds belonging to free chains in the solution.

The expression we have derived for $\Delta S_{1}$ is apparently in strong disagreement with the conclusions of de Gennes in reference [6], that $\Delta S_{1}=0$. This paper explains how to apply Edwards formalism [23] to the case of concentrated polymer systems trapped between two plates. The monomer concentration $c(z)$ is calculated from the relationship $c(z)=|\psi(z)|^{2}$, where $\psi$ is the local order parameter which is an eigenfunction of the Schrödinger equation. The solution $\psi(z)$ is then determined by the choice of boundary conditions. It is shown that the macroscopic boundary condition always appears for a concentrated system as a condition of zero slope. This is due to the exact compensation of the potential exerted directly by the wall on the monomers and the self-consistent potential associated with the monomer density variation near the surface. The density profile extends up to $\xi$, where $\xi$ is the correlation length of the polymeric system; in the case of a melt, $\xi=a$. The macroscopic boundary condition holds for $z>\xi$. As a consequence, the monomers located at $z>\xi$ are not aware of the existence of the surfaces, that cannot therefore interact with each other. (Up to this point, our work perfectly fits this more general theory). As a consequence, the free energy difference between the trapped polymer and the same amount of unconstrained chains is entirely located in the layers of thickness $\xi$ in contact with the solid surfaces. Counted by surface unit, $\Delta F$ is the surface energy $\gamma$. The main conclusion is the localization of $\gamma$ in the surface layer of thickness $\xi$ ( $\xi \rightarrow a$ for a melt). In general $\gamma$ contains enthalpic and entropic terms, but none of them comes from the bulk part of the slit. The conclusion $\Delta S_{1}=0$ of this paper should therefore be interpreted as concerning solely the bulk part of the chains, i.e. the segments located in the region $z>\xi$. In our paper, $\Delta S_{1}$ is the total entropy of confinement per chain, and it includes the surface layer contribution by taking into account the surface paths, which are actually the only contribution. The discrepancy with reference [6] thus appears as a matter of vocabulary, while the physical conclusions are clearly in good agreement.

From the present study, the surface energy $\gamma$ can be estimated for the melt in contact with a neutral wall :

$$
\gamma=-\frac{T \Delta S_{\mathrm{TOT}}}{\Sigma}
$$

where $\Sigma$ is the surface area of the solid.

$\Delta S_{\text {TOT }}$ is obtained by summing the contribution $\Delta s$ of all segments in the surface layer. In our case, we get :

$$
\Delta s=k_{\mathrm{B}} \ln \frac{4}{5}
$$

The number of segments per surface unit in the surface layer is $\frac{2}{3 a^{2}}$, hence :

$$
\gamma=-\frac{2}{3 a^{2}} k_{\mathrm{B}} T \ln \frac{4}{5} \simeq 0.15 \frac{k_{\mathrm{B}} T}{a^{2}} .
$$

However, this value depends on the choice of the lattice in several ways :

(i) through its coordination number $x$, 
(ii) through the choice of the cutting plane, which determines the surface coordination number $x-p$,

(iii) through the choice of the lattice parameter $a$. This point is difficult since $a$ should take care of the range of the monomer-monomer interaction because complete filling of the lattice is assumed, and on the other hand it should take care of the persistence length of the polymeric material because no correlation in the orientation of adjacent segments is considered.

The numerical value obtained for $\gamma$ has therefore no real physical meaning, and there is only little hope that such a calculation could be performed by the use of standard lattice models.

\section{End-to-end distance.}

As an important consequence of the present representation of polymer melts confined in slabs, the end to end distance of the polymer chains is expected to vary continuously with the distance $D$ between the walls. We have to emphasize that it is not a common statement. Our argument is the following : considering only the case where $a \ll D \ll N^{1 / 2} a$, the polymer chain is a succession of bulk and surface paths. Since the bulk path is 3-dimensional while the surface path is 2-dimensional, the projected (parallel to the walls) end to end distances of the two species differ by a constant. The projected end-to-end distance of the whole chain is obtained by adding the two contributions, and the result is therefore dependent on the distribution of the paths among the two kinds.

Using our previous results, and expanding the projected root mean square end-to-end distance $R_{\|}$at the first order in $\frac{a}{D}$, we get :

$$
R_{\|}=R_{\| \infty}\left(1+\frac{2}{3} \frac{a}{D}\right), \text { for } D \gg a
$$

where $R_{\| \infty}$ is the average end-to-end distance projected on the $x-y$ plane for the unperturbed ideal chain.

\section{Conclusion.}

All the results derived in the present study are based on the assumption that the surface layer and the bulk structures are uncoupled. This assumption is in complete agreement with the scaling description of the melt using a single correlation length of order $a$ [24] and is also supported by the recent Monte Carlo simulations of the polymer/solid interfaces [3,5].

Moreover it is in qualitative agreement with the computer calculations of Doros Theodorou [25] using the Scheutjens and Fleer [26] layered mean-field method.

However, some of the results obtained here are in obvious disagreement with some leitmotives of the polymer melt physics: this is the case for the dependence of the confinement entropy per chain with the distance $D$ between the plates, for the variation of the end-to-end distance of the trapped chains with $D$, or for the equivalence underlined between the confined melt with neutral plates and the confined ideal chains trapped between adsorbing plates ; actually, it is suggested in the present work that the term ideal chain which is used frequently in both problems has not exactly the same meaning in the melt and in the solution. Although the proposed picture can only be considered at this point as a conjecture [27], it could hopefully help in a concrete approach to a large variety of problems, like adhesion or copolymer structures. Moreover, it seems possible to extend some of the features of our picture to confined semi-dilute solutions since they are made of an «ideal » arrangement of 
blobs [24]. This extension could perhaps help to improve the continuity between models for semi-dilute solutions and melts at interfaces.

\section{Acknowledgements.}

This work has highly benefited of long and frequent discussions with Pr. Sanat Kumar, and was carried out in parallel with his off-lattice Monte Carlo simulations in a very interacting mode. I am also very grateful to Pr. Gerrit Ten Brinke and Pr. Michele Vacatello for many helpful discussions, and to Pr. Khaled Abdel-Ghaffar for having probed the validity of some of the present results using the rigorous approach of statistical mathematics. At last, I am indebted to Pr. P. G. de Gennes for illuminating comments.

This work has been supported by I.B.M. France and the I.B.M. Almaden Research Center in San Jose, California. I wish to thank Dr. Do Yoon for his nice hospitality.

\section{References}

[1] Horn R. G. and IsraelachVili J. N., Macromolecules 21 (1988) 2836-2841.

[2] Montfort J. P., Hadziloannou G., J. Chem. Phys. 88 (1988) 7187.

[3] Kumar S. K., Vacatello M., Yoon D. Y., J. Chem. Phys. 89 (1988) 5206.

[4] Madden W. G., J. Chem. Phys. 87 (1987) 1405-1422.

[5] Ten Brinke G., Ausserré D., Hadziononnou G., J. Chem. Phys. 89 (1988) 4374.

[6] De Gennes P. G., C.R. Hebd. Acad. Sci. Paris 290 (1980) 509.

[7] De Gennes P. G., C.R. Hebd. Acad. Sci. Paris 305 (1987) 1181.

[8] Yoon D. Y. and Flory P. J., Macromolecules 17 (1984) 868-871.

[9] Helfand E., Macromolecules 9 (1976) 307-310 and 311-316.

[10] Asakura S. and Oosawa F., J. Chem. Phys. 22 (1954) 1255.

[11] Casassa E. F., J. Polym. Sci., part B, Polymer Lett. 5 (1967) 773-778.

[12] BARKeR J. A., I.B.M. Res. Report (1984), RJ 4206 (46284).

[13] Ausserré D., Hervet H., Rondelez F., J. Phys. France Lett. 69 (1985) L-235.

[14] Svetogorski D. A., J. Phys. France 48 (1987) 689-692.

[15] Nakanishi H. and Bub Lee S., J. Phys. France 49 (1988) 1295.

[16] Guttman C. M., di Marzio E. A., Hoffman J. D., Polymer 22 (1981) 1466-1479.

[17] di Marzio E. A., Guttman C. M., Polymer 21 (1980) 733-744.

[18] MANSField M. L., Macromolecules 21 (1988) 126-130.

[19] De Gennes P. G., Macromolecules 13 (1980) 1069-1080.

[20] Casassa E. F., J. Polym. Sci. : Polym. Symp. 72 (1985) 151-160.

[21] Bouchaud E., Thèse, Paris-Sud (1988).

[22] di Marzio E. A., Rubin R. J., J. Chem. Phys. 55 (1971) 4318-4336.

[23] Edwards S. F., Proc. Phys. Soc. London 85 (1965) 613.

[24] De Gennes P. G., Scaling Concepts in Polymer Physics (Cornell Univ. Press, London) 1979.

[25] Theodorou D., Macromolecules 21 (5) (1980) 1391-1436.

[26] See e.g. Scheutjens J. M. H. M. and Fleer G. J., Macromolecules 18 (1985) 1882.

[27] It should also be kept in mind that most of the present calculations are oversimplified. For example, it is implicitly assumed at several places that the average of products is well approximated by the product of averages. In our case, this procedure should mainly affect numerical prefactors. 